\title{
Product Advanced Design: A Cultural Intermediation Between Knowledge and Information
}

\section{Elisabetta Cianfanelli}

Università degli Studi di Firenze

elisabetta.cianfanelli@unifi.it

ORCID 0000-0003-0241-1826

\section{Maria Claudia Coppola}

Università degli Studi di Firenze

mariaclaudia.coppola@unifi.it

ORCID 0000-0003-3776-9860

\author{
Margherita Tufarelli \\ Università degli Studi di Firenze \\ margherita.tufarelli@unifi.it \\ ORCID 0000-0003-4824-6715
}

\begin{abstract}
Digital transformation (DX) drives transversal change, breaking disciplinary silos to transition to more sustainable paradigms through new ontological and epistemological frameworks. This has consequences on product design and development too: since DX concerns cultural and meaning shifts, it enhances product development as a high-intensity knowledge-based process. Thus, product design shifts into its "Advanced" stage, enacting transcendence and translation of different kinds of knowledge into future-oriented artefacts. This highlights new needs in the generation and transmission of Advanced Design knowledge stemming from future artefact production instances.

By focusing on recent challenges rising in product design and development, this paper aims to discuss the cultural intermediation enacted by Advanced Design knowledge through the results of an applied research experience.
\end{abstract}

\section{Keywords}

Digital transformation

Product design

Product development

Knowledge management Design knowledge 


\section{Transformation}

Contemporary landscape could be compared to a constellation of radical interdependencies, entanglements, and relationships that challenge designers to focus on complex socio-technical systems to better support values such as equality and justice for both humans and nonhumans (Escobar, 2018).

Today, technology permeates every layer of society, engaging with networked subjects - beings and things - and objects material and digital artefacts - by mediating their mutual interactions. Here, technology is accelerating a deep transformation of the world as we are used to understanding it, raising the need for a new ontological and epistemological framework in many disciplines. What is commonly addressed as Digital Transformation (DX) is the result of the widespread adoption of technological tools, whose agency addresses the operational, organizational and managerial aspects of knowledge management, pushing for a shift of meaning and value production in many human activities (Epifani, 2020): design and production processes are in the line.

Such mediating technologies engage with cultural artefacts by gathering and collecting "such a quantity of objectified culture, as to make the subjective culture of those who use it appear inadequate" (Granelli, 2006). Whether people interact among themselves or with the rest of the world, it would happen through digital technologies, so that the constant is easily found: they enact a cultural mediation (Ferraris, 2021).

This sounds relevant to design, since it has always enabled a cultural mediation, negotiation and translation by itself, always enacting a synthesis of technological advancement, societal needs and market constraints: today DX reshapes industrial tools and processes, affecting design theory and practice. In fact, looking at the product design and development scenario, DX provides agile and real-time integrated environments, wherein design turns more digital than ever: the universal application of digital design package (e.g. CAD, FEA, CAE, CAM, etc.) speaks of a data-driven paradigm in manufacturing environments, directly affecting product design and development processes.

Since DX concerns cultural and meaning shifts, it enhances the "immaterial" side of product development, as it can be understood as a high-intensity knowledge-based process in need of better coordination and integration (Labbi \& Ahmadi, 2020). In fact, the success of product design and development in digital environments links to a massive information exchange. This is the reason behind the fast adoption of the advanced manufacturing technologies mentioned above, whose value is enhanced through coordination tools linking them all. Product Lifecycle Management (PLM) platforms in fact integrate all data collected during the steps of product development, enabling communication among all the players involved in the production chain, from designers to engineers, from commercials to logistics. Thus, product design shifts into its Advanced stage, resulting from the combination of project activities and digital tools organised according to participatory innovation processes (Labbi \& Ahmadi, 2020). 
By focusing on recent challenges rising in product design and development, this paper aims to outline how product design is surfing the troubled waters of the ongoing transformation. Even though technology winds blow hard for a fast-paced change, it seems that many disciplines are stuck in calm seas, with an old compass to show them the way.

Times of great change are waiting ahead: the need to transition to new cultural and economic paradigms is widely shared, although it is structurally hard to address. In its "dynamic waiting", design is trying to dispel such disorientation by seizing in this "middle sea" the opportunity to challenge and provoke current system foundations: by moving through a sort of "trans-temporality" between major historical events, heterogeneous sources of knowledge, and powerful yet partially unknown tools, design takes advantage of its usual "in-between" position to push a trans-disciplinary answer to change. Furthermore, the paper presents an applied research experience, in which design students were challenged to engage with the transition, transcendence and translation enacted by design through the development of future-oriented artefacts to ride the transformation. This highlighted, from an educational perspective, new needs in the transmission of knowledge for Advanced Design to ride the DX.

\section{Transition}

Today designers are challenged to conceive socio-designs for future environments that will be deeply changed by DX. As mentioned before, digital technologies enact the transformation of theoretical and operational spaces across all disciplines, so that the concept of "transition" is gaining an autonomous disciplinary thickness. The establishment of the New European Bauhaus movement for the Green Deal (Bason et al., 2020) makes "transition" strong enough to become a widespread cultural framework for many disciplines, design included: transitioning is a sort of commitment, a call to action stemming from the shared awareness of the unsustainability of some recent approaches, tools, and practices and their implications of the planet.

Even if design is well known for its ability to translate the technological advancement into things that people will love and use, it is becoming increasingly clear that a large part of these "innovative solutions" has contributed to building what the contemporary culture of transition wishes to tackle vigorously: designers are facing the critical aspects of their profession - which Papanek (1971) described as dangerous - especially when it becomes instrumental to the preservation of an economic and socio-technical model showing its structural issues.

Today's social, political, and environmental emergencies ask designers to enact new modes of theory and practice, shifting from their past de-futuring (Fry, 2010) action to a more teleological and committed one: essentially "what designers make becomes the future we inhabit" (Tonkinwise, 2015). The disciplinary contamination of design with future studies is not recent, but it seems to emerge in times of crisis, hence transition. The pandemic ranks 
among one of those, stimulating the need to think about alternative futures. Such shift in focus can be easily traced in humanistic production. Looking at designers, in fact, times of crisis and structural uncertainty turn them into visionaries at the service of society, "inventors of scenarios and strategies [who] must play in the territories of the imagination to create new stories, new fictions, which will add to the thickness of the real" (Branzi, 1999). Thus, design for transition is a design that makes an explicit commitment to society, furthering structural change in a proactive and synergic manner.

However, the exploration of possible futures is both a "trap and a trampoline" (Maldonado, 1971), because, although it embodies a radical research tool, it raises various challenges when the design process goes further in the translation of such vision into an artefact - whether tangible or intangible - , through which it should deliver that bit of future into everyday experience.

The need to explore and develop fictional scenarios seems to be the pivotal point on which research and practice hinge: the former needs it as an a priori exercise to generate new knowledge useful to frame problems; the latter needs it as a given context within which to apply that new knowledge to the design process. From a research perspective, the tension to futures links to a series of conceptual design practices (Dunne \& Raby, 2013) which registered growing interest in design schools as explicitly futures-oriented research methods. These approaches provide challenging exploratory devices for students, "as they allow the exercise of design to be removed from contextual and production constraints" (Quinz, 2020). However, as for practice, the resulting design outputs turn to be often artefact-totems (intentionally) distant from industrial reality, and, for this reason, they may activate potentially revolutionary conversations and provocations, while not triggering a concrete transition to alternative futures stemming from everyday life.

If it is true that "we are creating the world to be understood, not merely understanding the created world" (Holt, 2016), design for transition seems to inhabit two complementary times: on the one hand, a progressive present, in which design acts; on the other, a near-future towards which design directs its action.

\section{Transcendence}

Moving from different time dimensions, designers develop a number of approaches and tools whose adoption led to the sublimation of a third "middle" time, the Advanced Futures: "dormant" yet possible scenarios where design could engage with future behaviours, beliefs and lifestyles. Advanced Futures are infinite, as they embody the possibilities towards which the world could transition to. Since they are not predictions, they benefit from pluralistic contributions to turn their fictional intent into a consistent design opportunity: in the Advanced Futures designers gather different actors to co-find and co-frame possibilities, leading to the co-designing of new artifacts embodying new shared values.

These practices become relevant in relation to the hyper-volatilization of the market, in a measure that a "design without market" (Celaschi, 2015) becomes possible: here, the very first out- 
put to be designed - and, thus, to be researched through design is the market itself: the lack of traditionally intended demand makes the designer the self-producer of his own brief and of the requirements that define the project. Thus, the designer seems to play a strategic role of aggregation around the autonomous observation of needs that have not yet been "filtered by the market nor by entrepreneurs that [usually] commission their resolution and transformation in goods or services" (Celaschi, 2015).

Designing in the Advanced Future implies that even the design brief undergoes a designing intention. In this sense, it strongly recalls the "provotype" (Mogensen, 1992), a design artefact through which the project activities seek to understand and challenge stakeholder conceptions to generate alternative design concepts (Boer et al., 2013): in fact, while prototypes - design devices par excellence - are effective in reiterating solutions to problems already framed, "provotypes can be massively useful when we need to reach a little further in the future - or explore and break boundaries" (Boer et al., 2013). Originally developed for computational systems designers, provotypes bridge analysis and design, current practice and future change. By addressing the contradiction between tradition and transcendence tensions inherent to artefacts, provotypes focus on the skills owned by designers while conducting research in the present compared to those needed to operate effectively in the upcoming systems (Boer et al., 2013).

Today the evaluation of such balance should take into account the effects of DX on industrial environments: digital tools enter product development processes, generating opportunities for new interactions and relationships between the players of the productive chain (Cantamessa et al., 2020). Here, designers stumble on new management tools, which are cultural products (Ferraris, 2021), entering all the steps of the product design process. For a more detailed overview, technology is likely to play a twofold role: on one side, technology as an object enters the design process as a tool linked to optimization and evaluation; on the other side, technology as a subject enters the design process as a functional component, ensuring cutting-edge performance (Rampino, 2012). Such layered activities happen in a collaborative environment, whose success depends on alignment and coordination. By sharing and exchanging technical information, intermediation becomes the strategic answer to knowledge management. Although intermediation is a manyfold process, two major complementary stages seem to engage in product development: on one side, the intermediation by technology coordinates information, performance and feedback; on the other side the intermediation by design integrates visions, knowledge and experience.

Design, moving from the progressive present to the near future, crosses Advanced Futures, enacting a transcendence of knowledge and information through the combination of traditional and digital tools, diverging approaches towards possibilities and converging methods towards certainties. In this sense, intermediation by design starts with a provotype challenging conceptions and perceptions about values and market, while intermediation by technology builds the path toward feasibility, performance and delivery. 


\section{Translation}

The interplay between the two stages of intermediation leads to the translation of a vision into a contingent reality, stemming from the synthesis of a "puzzled knowledge".

Good quality of such intermediation activities rely on an equally good knowledge transfer, exchange and alignment. Digital technologies such as PLM platforms facilitate the management of different kinds of information. However, since knowledge application is linked to meaning and value generation, a cultural intermediation is needed and design seems to be in an ideal position in doing so, since design culture itself stems from deep interdependencies, contingencies and negotiations among different cultures: in this way design culture acts as a tool itself (Julier et al., 2019) catalyzing and emphasizing the pluralistic feature of designing environments. Cultural intermediation seems to be the core feature of design's strategic value within the advanced industry environments, in which the Advanced Product takes the shape of a co-designed unicum, an artefact where product and information merge, resulting in a synthesis of contributions coming from both humans and machines.

The arguments built so far have been the experimental foundation within which the Course of Product Advanced Design 2020/2021 from the University of Florence launched its designing challenge to the students. This edition of the course has been interpreted through the lens of the "trans-temporality" dimension and sought to ride the ongoing transformation by adopting a PLM as both a designing tool and an educational one.

The first goal in designing the course itself was to simulate an advanced product development environment, so that two enterprises - Savio Firmino and Edra - were involved in the design challenge, as well as students and professors coming from both design and engineering departments.

The class was challenged to investigate the Advanced - industrial - Design, interpreted as an advanced stage of made in Italy production, which could find in the latest technological trend a strategic opportunity. Here, design has been the object of inquiry and the tool for testing and evaluating how knowledge management technologies enter the design process and, in turn, what kind of further knowledge they produce or need.

Starting from the transformation background, the designing theme was about the smart chair and its next technological, aesthetic and experimental turn. The "chair" has always been an archetype in design culture, so that it could embody the perfect provotype on which different actors could discuss the future instances of living, starting from a semantic investigation on the "seat" Fig. 2 and the "seating" Fig. 3 to explore future working and relaxing habits. 

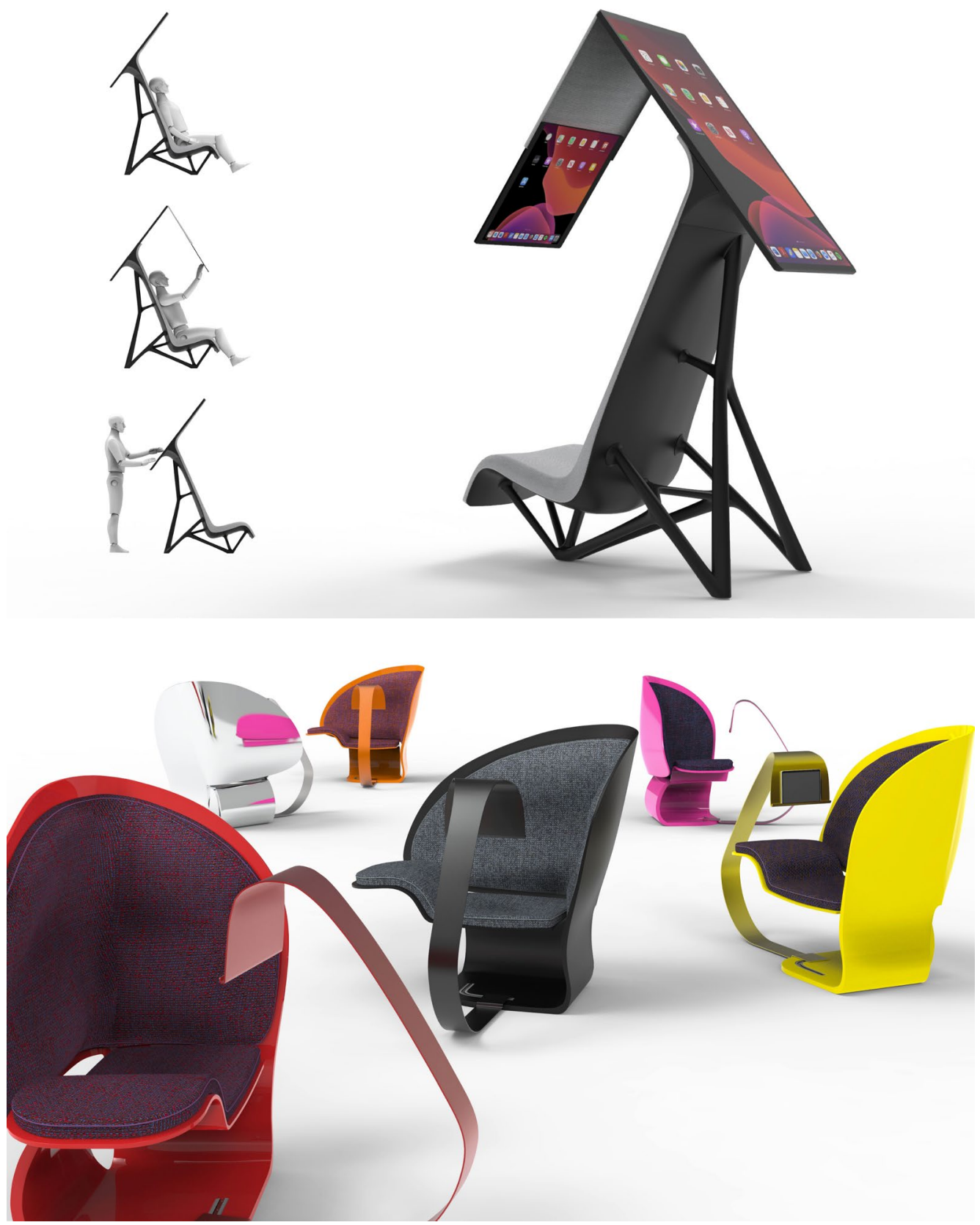

Fig. 1

Cirulli, A., Corenich, L.,

Di lenno, S., \& Silverii, A. (2021). HAL. Generative design process. REI Design Lab.

Fig. 2

Vannucci, A., Mati, G.,

Capaccio, L., \& Ferrari, V. (2021). TuliPOP chair. REI Design Lab. 
More specifically, the provotype became the object of the first analytic steps: the main goal was co-finding plausible narratives about a society set in a quite distant future - e.g. 2030-2050. The participatory exploration of Advanced Futures brought out challenging yet coherent visions about future behaviours, lifestyles and environments. These provided strategic information to students and enterprises: the first gathered information to design their own brief and project requirements; the latter engaged with the radical design of a market opportunity.

Once established a common ground on the provotype, designers could go further in the product design process, adopting the management tools inside the PLM platform to track process progression while communicating with other players of the chain. From preliminary sketches, to components engineering and assembly, from filling Bill of Materials to defining sourcing, production, distribution and communication strategies, from designing the market launch to the retail experience: designers have been challenged to deal with all the product development steps while adopting powerful tools of chain integration, playing a role of inter-/trans-disciplinary mediation.

The applied research experience presented here shows how tools gain a specific relevance in the design process, as they activate the inherent combinatorial side of design practice. However, although tools can be understood as just alternative resources which can be employed - or not - in the design process, digital technologies seem to make an exception, since they are tools enabling a combinatorial dimension by themselves, just like design does. The overlapping of these two kinds of intermediation shifts the focus of discussion toward the object of such a combinatorial activity: knowledge and information flows intermediated by both design and technologies.

\section{Transmission}

DX is driving horizontal change, breaking disciplinary silos and outlining new opportunities to transition to more sustainable paradigms. The radical change is pushing forward unprecedented domains of possibilities in daily practices, promoting new modes of doing and being. Such a societal transformation makes industrial design aware of the need to address all those "unresolved aspects that hinder a clear definition of [its] role [...] in society" (Maldonado, 1974).

While facing the uncertainty of a troubled tomorrow, design grows as a conversation about possibilities, so that it undergoes an ontological turn focusing on "understanding the dynamic designing relations between the world, things and human beings" (Fry, 2017) to foster pluriversal practices (Escobar, 2018). Today, the thoroughly constructed environment where humans and non-humans live embodies the formula "design designs", for as long as we design our world, our world designs us back (Willis, 2006), asking for new knowledge to enact designing intentions, provocations, and solutions. 
Designing certain digital tools - e.g. PLM platforms, generative algorithms, digital twins and simulation apps - and bringing them into the design process enabled the generation of new knowledge to address transition. The nature of such knowledge is challenging as it seems to be embedded in the intermediation in action throughout the whole product design and development process, while it develops through interfaces, digital workspaces and computational environments. If intermediation embodies the pivotal point in Advanced Product design and development, which bridges knowledge coming from players and tools, rather than players and tools themselves, then this raises questions about how design knowledge undergoes such intermediation and what will be the core skills to allow the transmission of such layered knowledge to strategic gatekeepers in the form of usable values.

The applied research experience highlights the situated relevance that tools gain while adopted in and adapted to a designing process or scope. On one hand, intermediation by technology boosts operational efficiency in terms of making available the latest version of product information to all the players in real-time - and sometimes even near-time - , it seems unsuitable to treat design knowledge as a whole. On the other hand, the inherent value of cultural intermediation by design, which holds together different kinds of knowledge, lies in the synthesis between scientific knowledge - what is measurable and explicit - and humanistic knowledge what is contingent and implicit.

As long as digital environments create the conditions for teamwork encouraging mutual cultural contamination among the members - even when they are all designers - , those places seem to have been designed to enhance design as a cultural mediator, rather than a technological one.

In fact, due to its specifically non-specific nature, design is not an autonomous discipline, but is traditionally influenced by a large number of external factors: as a heteronomous discipline, design feeds naturally from external knowledge in order to survive (Riccini, 2017). In digital environments this happens with ease to the point that during the "digestion" of such heterogeneous resources, design produces new mediated knowledge which is not specific to any of the swallowed disciplines and is contingent to the issues to be addressed. The resulting cultural package embedded in the design solution will then enable the materialization of fragments of change coming from Advanced Futures. This is the main reason why today, despite intermediation by technology manages strategic data and technical information through powerful tools, an intermediation by design seems to be necessary in the making use of available knowledge.

Advanced Design knowledge shifts its application becoming a resource and a utility (Drucker, 1993), namely an adaptive, collective, organized and transmissible one. Here, intermediation by design revolves around selecting, prioritizing and interpreting available knowledge, being it raw or processed: then, transmission will concern how designers pack the cultural framework where design practice will take place and how they deliver it in future artefact production instances.
Elisabetta Cianfanelli Industrial Design Specialist, Full Professor at DIDA (Department of Architecture) of Università degli Studi di Firenze (Italy), Design

Campus section. President of the Master Degree in Fashion System Design, and scientific director of the DIDA Lab REI (Reverse Engineering \& Interaction Design). Her research focuses on SMEs manufacturing environments and product design.

\section{Margherita Tufarelli \\ Designer, Ph.D. in Design. Currently a Research Fellow at DIDA (Depart- ment of Architecture) of the Università degli Studi di Firenze (Italy), Design Campus section. She is currently working on a research project founded by Regione Toscana. Her research interests concern the design-driven interplay between cultural heritage and digital technologies.}

\section{Maria Claudia Coppola} Ph.D. student in Design at Università degli Studi di Firenze, DIDA (Department of Architecture), Design Campus section and member of the DIDA Lab REI (Reverse Engineering \& Interaction Design). Digital culture enthusiast, her research combines design approaches with theories from social and political sciences to foster alternative public innovation models. 


\section{References}

Bason, C., Conway, R., Hill, D., \& Mazzucato, M. (2020). A New Bauhaus for a Green Deal. UCL London. https:// www.ucl.ac.uk/bartlett/ public-purpose/publications/2021/jan/new-bauhaus-green-deal

Boer, L., Donovan, J., \& Buur, J. (2013). Challenging industry conceptions with provotypes. CoDesign, 9(2), 73-89.

Branzi, A. (1999). La casa calda: esperienze del nuovo design italiano. Idea Books.

Cantamessa, M., Montagna, F., Altavilla, S., \& Casagrande-Seretti, A. (2020). Data-driven design: the new challenges of digitalization on product design and development. Design Science, 6 .

Celaschi, F. (2015). AdvanceDesign Points of View. In M. Celi (Ed.), Advanced Design Cultures (pp. 3-17) Springer.
Drucker, P.F. (1993). The rise of the knowledge society. The Wilson Quarterly, 17(2), 52-72.

Dunne, A., \& Raby, F. (2013). Speculative everything: design, fiction, and social dreaming. MIT Press.

Escobar, A. (2018).

Designs for the pluriverse:

Radical interdependence, autonomy, and the making of worlds. Duke University Press.

Epifani, S. (2020). Sostenibilità digitale: Perchè la sostenibilità non può prescindere dalla trasformazione digitale. Digital Transformation Institute.

Ferraris, M. (2021).

Documanità: Filosofia del mondo nuovo. Gius. Laterza \& Figli.

Fry, T. (2010). Design as politics. Berg.

Fry, T. (2017). Design for/by "The Global South". Design Philosophy Papers, 15(1), 3-37.
Granelli, A. (2006). I/ sé digitale. Identità, memoria, relazioni nell'era della rete. Guerini e Associati.

Holt, M. (2016). Baudrillard and the Bauhaus: The Political Economy of Design. Design Issues, 32(3), 55-66.

Julier, G., Folkmann, M. N., Skou, N. P., Jensen, H. C., \& Munch, A. V. (Eds.). (2019). Design Culture: objects and approaches. Bloomsbury Publishing.

Labbi, O., \& Ahmadi, A. (2020, March). PLM and Smart Technologies for Product and Supply Chain Design. In International Conference on Artificial Intelligence \& Industrial Applications (pp. 149-160). Springer.

Maldonado, T. (1971).

La speranza progettuale. Ambiente e società. Einaudi Editore.

Maldonado, T. (1974).

Avanguardia e razionalità: articoli, saggi, pamphlets 1946-1974. Einaudi Editore.
Mogensen, P. H. (1992). Towards a provotyping approach in systems development. DAIMI Report Series, 21(412).

Quinz, E. (2020). Contro l'oggetto, Conversazioni sul design. Quodlibet.

Papanek, V. (1971). Design for the real world. Human Ecology and Social Change. Thames \& Hudson.

Rampino, L. (2012). Dare forma e senso ai prodotti. II contributo del design al processo innovativo. Franco Angeli.

Riccini, R. (Ed.). (2017). Fare Ricerca in Design. II Poligrafo.

Tonkinwise, C. (2015). Design for Transitions-from and to what? Design Philosophy Papers, 13(1), 85-92.

Willis, A. M. (2006). Ontological designing. Design philosophy papers, 4(2), 69-92. 